\title{
Application Research on Transient Rayleigh Technology in Pile Foundation Detection
}

\author{
Wei Wu \\ Department of Architectural \\ Engineering \\ Beijing Polytechnic College \\ Beijing, China \\ weiwu1976@126.com
}

\author{
Jin Yang \\ School of Geophysics and Information \\ Technology \\ China University of Geosciences \\ (Beijing) \\ Beijing, China \\ yang_jin@163.com
}

\author{
Xianmin Zhang \\ Airpot College \\ Civil Aviation University of China \\ Tianjin, China \\ xmzhang@cauc.edu.cn
}

\begin{abstract}
Detection of pile foundation is one of the most important procedures in the evaluation of pile bearing capacity and pile quality. In this paper, the application of the transient Rayleigh technology in detection of pile foundation (pile integrity, pile verticality and pile bearing capacity) have been illustrated by few examples. By using transient Rayleigh technology, the shear wave velocity of soil around pile can be tested. The pile integrity, the pile verticality and the pile bearing capacity can also be detected rapidly. Comparing with the static load test and others dynamic technologies, this method is more economical and nondestructive.
\end{abstract}

Keywords-transient rayleigh technology; pile foundation detection; pile bearing capacity; share wave velocity; pile integrity ; pile verticality

\section{INTRODUCTION}

As one of the most commonly used foundation forms, pile foundation is an concealed structure. The quality of pile foundation is directly related to the operation and safety of the main structure. Once an accident happens in pile foundation, the reinforcement treatment is very difficult. Pile testing is an important measure to conduct a comprehensive evaluation on the pile bearing capacity and the pile quality.

At present, the main technologies used in pile foundation detection include: the direct methods such as static load test, drill core test, and indirect methods such as high strain method, low strain method, sound wave transmission method, etc. Static load test is more effective but it takes a long time and large investment, and in some cases it is difficult to achieve. Dynamic measurement technology has been applied widely, but it is very sophisticated to interpret in theoretical basis and testing technology and application, and needs further research.

Developed in the 1990' s, transient Rayleigh wave exploration technique is a new engineering and environmental geophysical method. By using the dispersion characteristics of Rayleigh wave and the relativity between wave velocity and physical mechanical character of site soil, many geological engineering problems could be solved. During the process of pile testing, transient Rayleigh wave technology could also play a very significant role.

\section{THEORY OF TRANSIENT RAYLEIGH WAVE TECHNOLOGY}

Surface waves are divided into Rayleigh and Love wave. Rayleigh wave has the strongest energy, the maximum amplitude, and the minimum frequency, making it easy to identify and measure.

The essence of transient Rayleigh exploration method is basing on the measurement of velocity of different frequency of Rayleigh wave to infer the rock and soil properties of the different depth (distance). During the detection process, an instantaneous impact force is exerted on the ground, and the Rayleigh waves with certain frequency range will be produced. The Rayleigh waves with different frequency will be superimposed together, and propagate forward in the form of pulse. Along the direction of wave propagation, $\mathrm{N}+1$ detectors (12 or 24) are set in $\Delta x$ interval, propagation characteristics of the Rayleigh wave of $\mathrm{N}^{*} \Delta \mathrm{x}$ length range could be tested. With the spectrum analysis, Rayleigh wave in each frequency can be isolated and one or more dispersion curves could be calculated. This multichannel superposition method could eliminate the random interference signal, and the geologic bodies in the dispersion curve reflects more prominent. This method has advantages of high construction efficiency, portable equipment and low cost. The factors that influenced the accuracy of longitudinal wave velocity and shear wave velocity are the extraction of Rayleigh wave, the computation of dispersion curves, and the inversion calculation of the longitudinal and shear wave velocity.

At present, f-k transform is widely used. The procedure is real amplitude recovery-spectrum analysis-1-D filtering-f-k transform-dispersion curves-interval velocity inversion. This method requires at least six common shot records to obtain a dispersion curve. The obtained dispersion curve is the weighted average level of the whole array length along horizontal direction. This method reduces the vertical and lateral resolution of Rayleigh wave, and some small local anomalies could not be detected. It is difficult to meet the accuracy requirements of detection.

To solve this problem, Professor Jiangping Liu proposed the adjacent-channel transient Rayleigh wave method that uses $\tau-p$ transform to extract the Rayleigh wave. The procedure is true amplitude recovery-spectrum analysis- ${ }^{\tau-p}$ filtering - 
Rayleigh wave extraction-calculation of dispersion curve of neighboring traces-interval velocity inversion. This method could eliminate the average effect and improve the vertical and lateral resolution of Rayleigh wave. It broaden the scope of application of transient Rayleigh wave method. Transient Rayleigh wave technology in pile foundation detection process can be used for pile integrity testing, pile verticality detection and single pile bearing force detection.

\section{Application of TransiEnt RAYLEIGH WAVE TECHNOLOGY IN THE DETECTION OF PILE FOUNDATION}

\section{A. Pile Integrity Detection}

Pile integrity refers to the pile conditions such as defects of segregation, necking, pile breaking, sediment in pile tip, and pile head laitance. It is a comprehensive qualitative index that could reflect the relative change of pile size, material compactness and continuity of the pile, which is classified by the effects on the bearing capacity of pile. Powder jet pile foundation is used widely in expressway construction. There is a direct drilling on soft soil foundation and the cement powder is evenly sprayed into the loose soil, which forms a semi rigid pile. It could reinforce foundation and prevent significant subsidence. Because its construction process is completed underground, making the detection of their quality is very difficult. The existing methods failed to achieve the purpose of construction quality control, therefore, the detection problem has been the "black box" problem.

Professor Jiangping Liu used the adjacent-channel transient Rayleigh wave method to detect the quality of cement injection pile. Wenxia Ding used this method to detect the quality of cement mixing pile. This technology improves the nondestructive detection precision, resolution, and detection efficiency. Mathematical models between velocity and physical properties have been bulit, and physical properties of pile could be obtained.

This method has following advantages: (1) The length, integrity and verticality of pile could be presented visually; (2) Without disturbing or damaging of the pile, the visual color classification of intensity could be obtained by quantitative processing of the wave velocity; (3) The broken piles and the strength of the weaker parts can be found in early stage; (4) Comparing with the static load test, the bearing capacity of the pile can be estimated; (5)The cost of testing can be reduced and the detection rate can be improved greatly.

\section{B. Pile Verticality Detection}

In ordinary industrial and civil construction, pile is generally designed to be a vertical pile. Nevertheless, the extrusion during construction, overload of bottom face in some area, horizontal displacement of supporting structure of foundation pit would lead to the deviation of pile and pile tilt. The stake inclination makes the pile under eccentric compression, reduces the bearing capacity, and breaks the pile. Therefore, the allowable deviation of pile verticality could not exceed $1 \%$ which is clearly stipulated in Code for Acceptance of Construction Quality of Building Foundation (GB502022002).

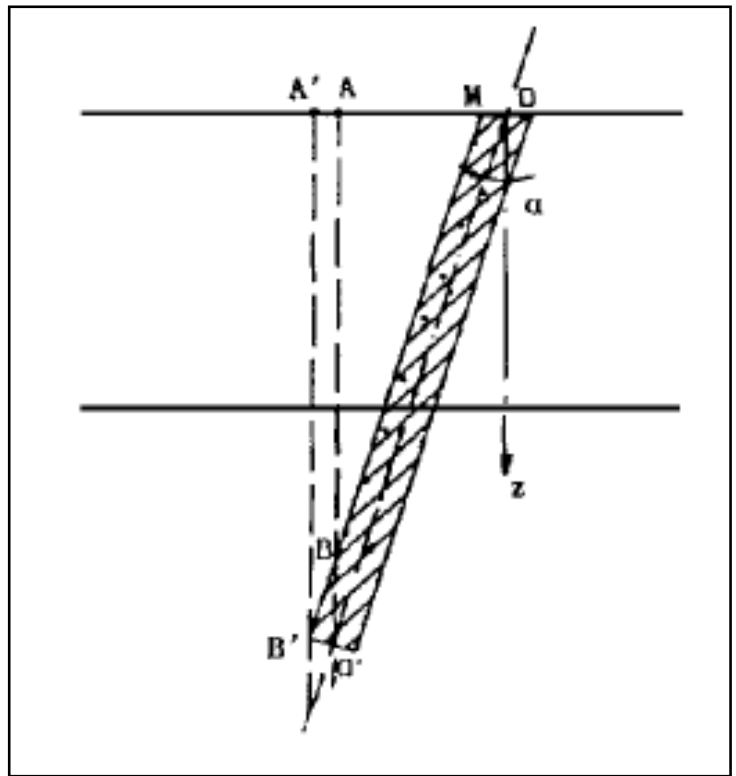

Fig. 1. Diagram of Detection Principle

The existing detection techniques of pile verticality is not very effectual, and the pile verticality detection has been a difficult problem in engineering field. With the progress of the detection technique of pile foundation, it is possible to test the actual verticality and angle of inclination of pile. Professor Yanhui Li put forward the method that uses Rayleigh wave to detect pile verticality based on the frequency dispersion of Rayleigh wave in the layered medium.

The dispersion curve tested by Rayleigh wave has an internal relation with underground medium structure. The stratigraphic structure within a certain depth of the ground can be received by inversion interpretation of dispersion curves. Since the propagation velocity of the Rayleigh wave in concrete is far greater than the propagation velocity of soil in surrounding soil, and the concrete density is higher than that of the surrounding soil density, it is easy to identify the interfaces of soil and concrete and to receive the interface burying depth of concrete. So the angle between pile and XOZ face could be calculated as :

$$
\alpha_{\mathrm{y}}=\tan ^{-1} \frac{M A}{A B}
$$

$\alpha_{\mathrm{x}}$ can also be calculated. Using the formula: $\cos ^{2} \alpha_{z}+\cos ^{2} \alpha_{x}+\cos ^{2} \alpha_{y}=2, \alpha_{z}$ could be derived. So the angle of inclination of pile is:

$$
\alpha=\frac{\pi}{2}-\alpha_{\mathrm{z}}
$$

By using this kind of test technology, a manual hole digging pile of high residence building was tested, and good results was achieved.

\section{Pile Bearing Capacity Detection}

Bearing capacity of single pile refers to the maximum external load that pile foundation could bear without stability failure and excessive settlement. There are many detection technologies which could be divided into two categories: direct 
method and indirect method. The stress of pile in static load test is close to its actual working condition, so the result is relatively reliable. But there are many deficiencies in test equipment, test efficiency, working conditions or expenses.

The author proposed an indirect method that Rayleigh wave was used to detect the bearing capacity of single pile. The model between shear wave velocity $\mathrm{v}_{\mathrm{s}}$ and pile side resistance $\mathrm{q}_{\text {sik }}$ and the model between shear wave velocity $\mathrm{v}_{\mathrm{s}}$ and pile tip resistance $\mathrm{q}_{\mathrm{pk}}$ could be obtained through SPT blow count $\mathrm{N}_{63.5}$. And the bearing capacity of single pile could be determined.

The relation between $\mathrm{N}_{63.5}$ and $\mathrm{q}_{\text {sik }}$ and the relation between $\mathrm{N}_{63.5}$ and $\mathrm{q}_{\mathrm{pk}}$ could_be derived using the hyperbolic function models (3). The asymptote of the formula could determine the limit value of soil strength. It not only considers the nonlinear characteristics of soil strength, but also reflects the limit value of the strength of the different soil types.

$$
q_{s i k}, q_{p k}=\operatorname{ath}\left(N_{63.5} / b+c\right)
$$

Equation (3), a, b, and c are undetermined coefficients, and could be determined by detection data of a lot of test piles.

$\mathrm{N}_{63.5}$ and $\mathrm{v}_{\mathrm{s}}$ could all reflect the soft and hard degree of soil layer, and the model between them could use exponential function ( $\left.v_{s}=a N_{63.5}^{b}\right)$. Considering the complexity of the region and classification of soil, the models from different soil properties of three different regions were chosen, and the models between $\mathrm{v}_{\mathrm{s}}$ and $\mathrm{q}_{\mathrm{sik}}$ and the models between $\mathrm{v}_{\mathrm{s}}$ and $\mathrm{q}_{\mathrm{pk}}$ have been built as:

$$
q_{s i k}, q_{p k}=\operatorname{Ath}\left(B \lg \mathrm{v}_{s}+C\right)
$$

With bored pile, for example, the $\mathrm{A}, \mathrm{B}$, and $\mathrm{C}$ value were shown in Tab. I. Once a pile is detected, the first step is to categorize the soil around the pile according to different soil property, the second step is to use the Rayleigh wave method to test the shear wave velocity of each layer, and the final step is to calculate $\mathrm{q}_{\mathrm{sik}}$ and $\mathrm{q}_{\mathrm{pk}}$ of each layer and determine the bearing capacity of single pile.

The pile foundation of one high-rise building was tested by this technology. By using the Model I, the result (Tab. II) showed that the ratio of calculated and static load results was between 0.7625 to 1.0072 , the average value was 0.8868 , and the standard deviation was 0.0942. By using the Model II, the ratio of calculated and static load results was between 0.7562 to 1.0111 , the average value was 0.8784 , and the standard deviation was 0.0910; By using the Model III, the ratio of calculated and static load results was between 0.7413 to 0.9874 , the average value was 0.8680 , and the standard deviation was 0.0920 . The results are secure and the accuracy of using the transient Rayleigh wave to test the bearing capacity of single pile is relatively high.

\section{CONCLUSION}

Using the transient Rayleigh wave detection technology to test pile foundation (including pile integrity, pile verticality, and bearing capacity of single pile ) have obvious advantages: (1) The length and integrity of pile can be tested without disturbing and destroying the pile body; (2) The verticality and pile inclination angle of pile body can be rapid detected ; (3) The bearing capacity of single pile could be estimated. This technology could reduce the cost, improve the efficiency, and have no damage. But more research works aiming at different regions, different type of soil, different type of pile should be done in future.

\section{REFERENCES}

[1] Xianhai Song, Duanyou Li, Rayleigh Wave Theory and Application China Water Power Press, 2010.

[2] Jiangping Liu, Weisheng Hou, Shunfang Xu, "Application of AdjacentChannel Transient Rayleigh Wave in Detection of Strength of Cutoff Wall,” Yangtze River, China, vol. 34, no. 2, pp. 35 36, 2003.

[3] Wenxia Ding, Qinghua Guo, "Application of Rayleigh Wave Method in Detection of Construction Quality of Cement Injection Pile," Hunan Agricultural Machinery, China, vol. 37, no. 1, pp. 58 60, 2010.

[4] Youyang Xin, "The Detection of Rayleigh Wave in the Application of Cement-Soil Mixing Pile," Low Temperature Architecture Technology, China, no. 1, pp.105 107, 2015

[5] Yanhui Li, Jiangping Liu, Xiankang Tan, "The Rayleigh Wave Method for Measuring the Slant Pile," Soil Engineering and Foundation, China, vol. 15, no.2, pp. 39 42, 2001.

[6] Wei Wu, Xianmin Zhang, Jin Yang, "The Application of Surface Wave Test Technique To Estimating the Bearing Capacity of Pile," Geophysical and Geochemical Exploration, China, vol. 28, no. 1, pp. 88 91, 2004.

[7] Junlong Liu, "Using SPT Blow Count to Estimate Ultimate Bearing Capacity of Single Pile." Geotechnical Engineering Technique, China, no. 2, pp. 88 91, 2000 
TABLE I. A, B, C VALUE OF Bored Pile

\begin{tabular}{|c|c|c|c|c|c|c|c|c|c|c|}
\hline & \multirow{2}{*}{ Soil Property } & \multicolumn{3}{|c|}{ Model I } & \multicolumn{3}{|c|}{ Model II } & \multicolumn{3}{|c|}{ Model III } \\
\hline & & $A$ & $B$ & $C$ & $A$ & $B$ & $C$ & $A$ & $B$ & $C$ \\
\hline \multirow{6}{*}{$\mathrm{q}_{\text {sik }}$} & Clay Soil & 98 & 1.0089 & -1.8475 & 98 & 1.4269 & -2.8162 & 98 & 1.2512 & -2.4525 \\
\hline & Brown Clay & 70 & 1.0089 & -1.8475 & 70 & 1.4269 & -2.8162 & 70 & 1.2512 & -2.4525 \\
\hline & Fine Sand & 80 & 1.1148 & -2.1139 & 80 & 0.8333 & -1.4629 & 80 & 1.001 & -1.8350 \\
\hline & Medium Sand & 90 & 1.1148 & -2.1139 & 90 & 0.8333 & -1.4629 & 90 & 1.001 & -1.8350 \\
\hline & Coarse Sand & 116 & 1.1148 & -2.1139 & 116 & 0.8333 & -1.4629 & 116 & 1.001 & -1.8350 \\
\hline & Gravel Sand & 135 & 1.1148 & -2.1139 & 135 & 0.8333 & -1.4629 & 135 & 1.001 & -1.8350 \\
\hline \multirow{6}{*}{$\mathrm{q}_{\mathrm{pk}}$} & Clay Soil & 1600 & 0.9685 & -1.9216 & 1600 & 1.3699 & -2.8515 & 1600 & 1.2012 & -2.5024 \\
\hline & Fine Silty Sand & 1100 & 1.3387 & -2.6707 & 1100 & 1 & -1.8895 & 1100 & 1.2012 & -2.3360 \\
\hline & Fine Sand & 1500 & 1.3387 & -2.6707 & 1500 & 1 & -1.8895 & 1500 & 1.2012 & -2.3360 \\
\hline & Medium Sand & 1900 & 1.3387 & -2.6707 & 1900 & 1 & -1.8895 & 1900 & 1.2012 & -2.3360 \\
\hline & Coarse Sand & 2500 & 1.3387 & -2.6707 & 2500 & 1 & -1.8895 & 2500 & 1.2012 & -2.3360 \\
\hline & Gravel Sand & 2500 & 1.3387 & -2.6707 & 2500 & 1 & -1.8895 & 2500 & 1.2012 & -2.3360 \\
\hline
\end{tabular}

TABLE II. COMPARISON RESUlts OF TRANSIENT RAYlEIGH WAVE METHOD AND STATIC LOAD TEST

\begin{tabular}{|c|c|c|c|c|c|c|c|c|}
\hline \multirow{2}{*}{ No. } & \multirow{2}{*}{ Length/m } & \multirow{2}{*}{\begin{tabular}{|c|} 
Static \\
Load \\
Test $/ \mathbf{k N}$ \\
\end{tabular}} & \multicolumn{2}{|c|}{ Model I } & \multicolumn{2}{|c|}{ Model II } & \multicolumn{2}{|c|}{ Model III } \\
\hline & & & $Q_{u k} / k N$ & $\alpha_{1}$ & $Q_{u k} / k N$ & $\alpha_{2}$ & $Q_{u k} / k N$ & $\alpha_{3}$ \\
\hline 1 & 28.9 & 3300 & 3256.35 & 0.9868 & 3148.42 & 0.9541 & 3165.80 & 0.9593 \\
\hline 3 & 33.5 & 4200 & 3711.40 & 0.8837 & 3643.40 & 0.8675 & 3650.99 & 0.8693 \\
\hline 4 & 28.9 & 3600 & 2745.06 & 0.7625 & 2722.25 & 0.7562 & 2668.69 & 0.7413 \\
\hline 5 & 33.5 & 3300 & 3323.89 & 1.0072 & 3336.78 & 1.0111 & 3258.36 & 0.9874 \\
\hline 6 & 28.9 & 3300 & 2787.6 & 0.8447 & 2806.63 & 0.8505 & 2737.85 & 0.8296 \\
\hline 7 & 33.5 & 3900 & 3259.22 & 0.8357 & 3241.72 & 0.8312 & 3201.33 & 0.8208 \\
\hline
\end{tabular}

